\title{
Os desafios da enfermagem obstétrica no início da pandemia da COVID-19 no
}

\section{Estado do Pará}

\author{
The challenges of obstetric nursing in the beginning of the COVID-19 pandemic in the state of Pará \\ Los retos de la enfermería obstétrica en el inicio de la pandemia COVID-19 en el estado de Pará
}

Recebido: 17/02/2021 | Revisado: 23/02/2021 | Aceito: 28/02/2021 | Publicado: 07/03/2021

Tais Pereira da Costa

ORCID: https://orcid.org/0000-0003-3568-3481

Universidade Federal do Pará, Brasil

E-mail: taiscosta93@gmail.com

Elisângela da Silva Ferreira

ORCID: https://orcid.org/0000-0002-2506-1622

Universidade Federal do Pará, Brasil

E-mail: licalipe8@yahoo.com.br

Diego Pereira Rodrigues

ORCID: https://orcid.org/0000-0001-8383-7663

Universidade Federal do Pará, Brasil

E-mail: diego.pereira.rodrigues@gmail.com

Débora Thalita Neri

ORCID: https://orcid.org/0000-0001-6658-2304

Centro Universitário Fibra, Brasil

E-mail: tathaneri@gmail.com

Elannira Amaral Soares

ORCID: https://orcid.org/0000-0003-4282-5994

Universidade Federal do Pará, Brasil

E-mail: nira_amaral@hotmail.com

Gabriela Campos de Freitas Ferreira

ORCID: https://orcid.org/0000-0002-7806-5280

Universidade Federal do Pará, Brasil

E-mail: freitas-gabi1@hotmail.com

Rafaela Moura de Araújo

ORCID: https://orcid.org/0000-0002-9793-7785

Universidade Federal do Pará, Brasil

E-mail: rafaela_3r@hotmail.com

\begin{abstract}
Resumo
Este estudo teve por objetivo relatar a experiência sobre os desafios da enfermagem obstétrica no contexto do início da pandemia da COVID-19 no Estado do Pará. Trata-se de um estudo descritivo do tipo relato de experiência realizado durante a pandemia do novo Coronavírus, sobre a atenção obstétrica prestada, dentro do período de março a agosto de 2020. Dentro dos resultados destaca-se que a partir dos boletins epidemiológicos, foi demonstrado que dentre os estados que mais concentraram óbitos de gestantes por COVID-19, o Pará ocupa o $6^{\circ}$ lugar com 08 casos registrados, até o dia 01/08/2020. Atuando no campo obstétrico durante a pandemia, nos meses iniciais e críticos da doença no Brasil, observaram-se várias dificuldades e entraves no fluxo e estrutura do atendimento obstétrico nos campos de atuação da enfermagem. Verificou-se inúmeros afastamentos de profissionais, por serem de grupo de risco, e até mesmo, a interrupção de atendimentos de pré-natal, com cancelamento das consultas, durante a fase complicada, de altos índices da doença. Deste modo, deve-se refletir os desafios que o profissional enfermeiro enfrentou e deve superar ao vivenciar a pandemia da COVID-19 no que diz respeito à sua assistência obstétrica.
\end{abstract}

Palavras-chave: Mortalidade materna; Infecções por coronavírus; Cuidados de enfermagem.

\section{Abstract}

This study aimed to report the experience on the obstetric nursing challenges in the context of the beginning of the COVID-19 pandemic in Pará, a state of Brazil. This is a descriptive study as an experience report carried out during the pandemic of the new Coronavirus, on the obstetric care provided, from March to August 2020. In the results, it is highlighted that from the epidemiological bulletins, it was demonstrated that among the states that most concentrated deaths of pregnant women due to COVID-19, Pará occupies the 6th place with 08 registered cases, until 08/01/2020. In the obstetric field during the pandemic, in the initial and critical months of the disease in Brazil, several difficulties and obstacles were observed in the flow and structure of obstetric care in the fields of nursing practice There were countless removals of professionals, as they are at risk, and even, the interruption of prenatal care with cancellation of 
consultations during the complicated phase of high rates of the disease. Thus, one must reflect the challenges that the nurse professional faced and must overcome when experiencing the COVID-19 pandemic with regard to their obstetric care.

Keywords: Maternal mortality; Coronavirus infections; Nursing care.

\section{Resumen}

El presente estudio tuvo como objetivo reportar la experiencia sobre los desafíos de la enfermería obstétrica en el contexto del inicio de la pandemia COVID-19 en el Estado de Pará. Se trata de un estudio descriptivo del tipo de experiencia realizada durante la pandemia del nuevo Coronavirus, sobre la atención obstétrica brindada, en el período de marzo a agosto de 2020. Dentro de los resultados, se destaca que a partir de los boletines epidemiológicos se determinó que entre los estados que más concentraron las muertes de gestantes por COVID-19, Pará ocupa el sexto lugar lugar con 08 casos registrados, hasta el 08/01/2020. Actuando en el campo obstétrico durante una pandemia, en los meses iniciales y criterios de la enfermedad en Brasil, se observaron varias dificultades y obstáculos en el flujo y estructura de la atención obstétrica en los campos del desempeño de enfermería. Hubo numerosos retiros de profesionales, por estar en riesgo, e incluso, una interrupción de la atención prenatal, con cancelación de consultas, en una fase complicada, con altas tasas de enfermedad. Por lo tanto, se deben reflejar los desafíos que el enfermero profesional enfrentó y debe superar al experimentar una pandemia de COVID-19 con respecto a su atención obstétrica.

Palabras clave: Mortalidade materna; Infecciones por coronavirus; Atención de enfermería.

\section{Introdução}

A Síndrome Respiratória Aguda Grave do Coronavírus 2 (SARS-CoV-2) é causada por um novo tipo de coronavírus que leva a uma doença infecciosa emergente com notável envolvimento pulmonar. Este vírus surgiu na China, em dezembro de 2019 e, desde então, espalhou-se por diversos países do mundo, tornando-se uma pandemia (Taisheng, 2020). A Organização Mundial de Saúde (OMS) nomeou a doença causada pelo novo vírus de COVID-19, sendo declarada uma emergência de saúde pública de importância internacional em 30 de janeiro de 2020, e em 11 de março de 2020 como uma pandemia (Brasil, 2020a).

Em abril de 2020, o Ministério da Saúde do Brasil incluiu todas as gestantes, puérperas e pacientes com perda gestacional ou fetal de até 15 dias no grupo de risco para COVID-19 (Takemoto et al., 2020).

As taxas de incidência da COVID-19 em gestantes, comporta-se de formas diferentes nos diversos países do mundo, com causas multifatoriais, sendo demarcadas por influências socioeconômicas e geográficas. A incidência e a mortalidade dessa infecção também são distintas nas diversas regiões do Brasil, incluindo a região Norte, em especial o estado do Pará. Nesse contexto torna-se extremamente importante a adequação da assistência obstétrica durante a pandemia COVID-19, oferecendo o cuidado baseado em evidências científicas, às gestantes e puérperas nas diversas fases da infecção, definindo diretrizes que evitem a morbimortalidade do binômio mãe-feto (Brasil, 2020a).

Enquanto residente de enfermagem obstétrica atuante no campo da assistência hospitalar e atenção primária, durante o início do período pandêmico, foi possível presenciar diversos problemas que impactaram diretamente na assistência obstétrica e, possivelmente, na mortalidade materna, o que resultou na motivação para realização desse estudo.

Diante das complicações que podem ocasionar à gestação e ao feto, faz-se necessário refletir e pesquisar sobre o ciclo gravídico/puerperal durante o período pandêmico da COVID-19, além de salientar a importância do cuidado profissional, sobretudo de enfermeiros, a fim de superar os inúmeros desafios que permeiam esse contexto. Uma vez que as informações são limitadas torna-se vital que os dados científicos e as experiências sobre a doença sejam compartilhados de maneira concisa e prática e a importância do enfermeiro diante do manejo e da assistência durante o período da pandemia seja demonstrado como uma das medidas para diminuição e/ou controle da mortalidade materna.

Nesse contexto, o presente estudo tem por objetivo relatar a experiência sobre os desafios da enfermagem obstétrica no contexto do início da pandemia da COVID-19 no estado do Pará. 


\section{Metodologia}

Trata-se de um estudo descritivo, qualitativo, do tipo relato de experiência realizado durante a pandemia do novo Coronavírus, sobre a atenção obstétrica prestada, dentro do período de março a agosto de 2020.

Relato de experiência é uma produção científica na modalidade qualitativa, valorizando o cultivo de conhecimentos, no qual o autor realiza a elaboração e reinscrição do tema através da memória, onde o sujeito constrói seus direcionamentos de pesquisa ao longo da evolução dos diferentes propósitos. Assim, o relato de experiência se enquadra como uma narrativa científica capaz de englobar produções e processos de caráter subjetivos. Essa produção científica se caracteriza também por uma pluralidade de bases teóricas e metodológicas, valorizando o sentido descritivo e interpretativo circunscrito em um determinado tempo histórico (Daltro \& Faria, 2019).

O Estudo foi realizado em uma maternidade de referência de alto risco obstétrico do estado do Pará e na rede de atenção básica no município de Belém-Pará, durante a atuação da pesquisadora enquanto residente em enfermagem obstétrica da Universidade Federal do Pará, no período deste estudo.

Por trata-se de um relato de experiência, sem a utilização de dados primários e pesquisas com seres humanos, o presente estudo não necessitou do Parecer de um Comitê de Ética em Pesquisa.

Para subsidiar esse estudo foram utilizados dados secundários publicados referentes às mortes maternas de Síndrome Respiratória Aguda Grave (SRAG) por COVID-19, ocorridas no Brasil e especificamente no Pará, através de Boletins Epidemiológicos Especiais pela Doença do Coronavírus - COVID-19 do Ministério da Saúde (MS) e da Secretaria de Saúde do Estado do Pará (SESPA).

Com base nesses dados foi realizada uma análise crítica reflexiva sobre a possível relação da mortalidade materna e a atenção obstétrica no início da pandemia da COVID-19 no estado do Pará.

\section{Resultados}

Durante o período marcado pela pandemia do novo Coronavírus, foram emitidos diversos boletins epidemiológicos, tanto a nível nacional, pela Secretaria de Vigilância em Saúde (Ministério da Saúde), como local (através das secretarias estaduais e municipais de saúde) demonstrando dados acerca de casos notificados, confirmados e mortalidade. Em alguns destes boletins contém dados de populações específicas, como de gestantes e puérperas.

Os dados utilizados neste estudo relacionados às mortes maternas no Brasil por COVID-19, compilados e descritos no Quadro 1, são segmentados em 42 semanas epidemiológicas (SE) e disponibilizados em 36 boletins. 
Quadro 1. Compilação dos Boletins Epidemiológicos Especiais - COVID-19, ressaltando casos de óbitos maternos, no período de janeiro a agosto de 2020.

\begin{tabular}{|c|c|}
\hline $\begin{array}{l}\text { Boletim } \\
\text { Epidemiológico } \\
\left(\mathbf{N}^{\mathbf{o}}\right)\end{array}$ & Informações COVID-19 e gestação \\
\hline $01-05$ & Não traz informações \\
\hline 06 & Inclui gestantes de alto risco como potenciais complicações ao COVID-19 \\
\hline 07 & Afastamento/mudança de função de profissionais do grupo de risco - gestantes de alto risco \\
\hline $08-13$ & Não traz informações \\
\hline 14 & $\begin{array}{l}\text { Distribuição de casos de SRAG segundo faixa etária e fatores de risco/comorbidades, incluindo os } \\
\text { casos confirmados de COVID-19, em gestantes/puérperas. }\end{array}$ \\
\hline 15 & $\begin{array}{l}\text { Traz informações da importância do histórico de vigilância da razão de mortalidade materna e inclui } \\
\text { pela primeira vez o registro de profissionais de saúde. }\end{array}$ \\
\hline 16 & Não traz informações \\
\hline $\begin{array}{l}17(\mathrm{SE} 21-17 \text { a } \\
23 / 05 / 20)\end{array}$ & $\begin{array}{l}\text { Registrados } 36 \text { óbitos maternos. Sendo } 7 \text { casos por COVID-19 ocorridos na região Norte, destes } 2 \\
\text { óbitos de gestantes registrados no Pará. } \\
\text { Descrito, pela primeira vez, o perfil de gestantes acometidas por COVID-19. }\end{array}$ \\
\hline $18-20$ & Não traz informações \\
\hline $\begin{array}{l}21(\text { SE } 27- \\
28 / 06 \text { à } \\
04 / 07 / 2020)\end{array}$ & $\begin{array}{l}\text { Registrados } 93 \text { óbitos maternos, sendo } 17 \text { casos por COVID-19 ocorridos na região Norte, destes } 5 \\
\text { óbitos de gestantes registrados no estado do Pará. }\end{array}$ \\
\hline $22-24$ & Não traz informações \\
\hline $\begin{array}{l}25(\text { SE } 31- \\
26 / 07 \text { a } \\
01 / 08 / 2020)\end{array}$ & $\begin{array}{l}\text { Registro de } 135 \text { óbitos maternos, sendo } 23 \text { óbitos de gestante com SRAG por COVID-19 no Norte. } \\
\text { Destas, } 08 \text { casos no estado do Pará. }\end{array}$ \\
\hline $26-36$ & Não traz informações \\
\hline
\end{tabular}

Fonte: Ministério da Saúde (2020).

Sobre a situação obstétrica, os últimos registros de óbitos maternos, dentro do período do estudo, ocorreram no BE 25 (Quadro 1), na semana epidemiológica 31, no período de 26/07/2020 à 01/08/2020. Os dados demonstraram que a faixa etária de 30 a 39 anos é a mais acometida, com 64 (32,2\%) óbitos; raça/cor mais frequente parda (50,4\%), mais da metade das gestantes $(56,3 \%)$ estavam no $3^{\circ}$ trimestre de gestação, e a maioria tinha grau de escolaridade até o ensino médio (25,2\%), 65 $(48,1 \%)$ apresentaram pelo menos um fator de risco ou comorbidade associada. Destas, 74 (54,8\%) foram internadas em Unidade de Terapia Intensiva (UTI) e, 54 (73,0\%) fizeram uso de suporte ventilatório invasivo.

Até o início de agosto de 2020, dentre os estados que mais concentraram óbitos de gestantes por COVID-19, o Pará ocupava o $6^{\circ}$ lugar com 08 casos registrados. Entretanto, dados fornecidos pela Secretaria de Saúde do Estado do Pará (SESPA), evidenciaram um total de 182 casos de COVID-19 em gestantes, com índice de 17 óbitos, até a data de 03/07/2020 (Quadro 1). Todavia, é notadamente importante destacar que tais dados incluem puérperas e possui o Sistema de Monitoramento de COVID-19 da SESPA como fonte dos dados, diferentemente ao do MS que possui como procedência o Sistema de Informação de Vigilância Epidemiológica da Gripe - SIVEP Gripe e inclui apenas gestantes.

Atuando no campo obstétrico durante a pandemia, nos meses iniciais e críticos da doença no Brasil, observou-se 
várias dificuldades e entraves no fluxo e estrutura do atendimento obstétrico nos campos de atuação da enfermagem.

Inicialmente, percebeu-se que em algumas unidades básicas de saúde do município de Belém, não houve mudança na organização do fluxo para distanciamento de pacientes sintomáticas, e sem disponibilidade de testes para detecção da COVID19 a serem realizados nas UBS. O mesmo ocorreu no âmbito hospitalar, que além disso, recebeu gestantes referenciadas de diversos municípios do estado, muitas vezes em estado grave e/ou avançado da SRAG.

Durante esse período inicial, verificou-se inúmeros afastamentos de profissionais, por estarem incluídos nos grupos de risco, tanto na atenção básica quanto no hospital. Além disso, o receio e medo, por parte dos profissionais de saúde, quanto à propagação do vírus nesses ambientes e risco de contágio e infecção, culminaram em cancelamento de consultas de pré-natal e cadastro de novas gestantes no Programa.

Com o registro de novos casos e publicação de estudos sobre a infecção pelo Coronavírus em gestantes e puérperas, além de orientações das autoridades sobre o distanciamento social e lockdown em várias cidades no Brasil, incluindo Belém e outros municípios do estado do Pará, surgiram portarias e manuais pelo Ministério da Saúde e secretarias do estado e municípios que impulsionaram diversas medidas para prevenção de contaminação e propagação do Coronavírus nos estabelecimentos de saúde.

No âmbito da Atenção Básica, após essas publicações, não foram observadas grandes mudanças, tanto em relação de infraestrutura, quanto à manutenção de consultas e atividades realizadas pelos enfermeiros. As consultas de enfermagem no Programa de Pré-natal continuaram suspensas e as orientações quanto aos recursos físicos e humanos não foram seguidos.

Apesar de não ter observado, inicialmente, um cumprimento por parte da Atenção Básica às recomendações dos órgãos mencionados, a maternidade de referência materno-infantil do estado do Pará adotou diversas estratégias para minimizar os riscos de propagação da doença às gestantes, puérperas e profissionais da saúde.

$\mathrm{Na}$ triagem obstétrica criou-se um anexo para gestantes que apresentavam sinais e sintomas de síndrome gripal, juntamente com a restrição de acompanhantes. Além disso, diversas enfermarias foram ajustadas para receber gestantes e puérperas suspeitas ou diagnosticadas com COVID-19. Dentro desse panorama, destaca-se ainda a orientação da Comissão de Controle de Infecção Hospitalar (CCIH) da Instituição, para que houvesse a intensificação do uso dos Equipamentos de Proteção Individual (EPI) para pacientes e profissionais.

Alguns boletins epidemiológicos demonstram os altos índices de profissionais da saúde notificados/confirmados com SRAG por COVID-19, estando em primeiro lugar os profissionais da enfermagem, primeiramente o técnico/auxiliar de enfermagem, seguido pelo enfermeiro. Diante disso, fez-se necessária a reorganização do serviço, entretanto, o acompanhamento pré-natal e o serviço de planejamento familiar foram suspensos em alguns serviços de saúde. Vale ressaltar que essa medida não seguiu as recomendações e orientações fornecidas pelos órgãos do governo estadual e federal.

\section{Discussão}

Diante do elevado número de casos no início da pandemia faz-se necessário refletirmos sobre a atenção obstétrica, mais especificamente quanto ao cuidado de enfermagem, especialmente no início da pandemia do COVID-19.

Pesquisas recentes demonstraram que a gravidez e o puerpério apresentam riscos adicionais para gestantes, puérperas e recém-nascidos com COVID-19 (Takemoto et al., 2020). Para esses autores, devido a testagem em grávidas ser obrigatória apenas em casos de gravidade, certamente há subnotificação dos casos confirmados. Portanto, acredita-se que os números de casos suspeitos/confirmados ou da mortalidade materna dentro do País e/ou do Estado do Pará podem ser maiores do que os divulgados pelo Ministério da Saúde e pelas Secretarias de Saúde.

As diferenças nos números de testes realizados por milhão de habitantes entre os países refletem magnitudes diferentes de subnotificação e os dados de estudos realizados reforçam a necessidade da testagem universal de pacientes 
obstétricas como uma estratégia urgente para a proteção de mulheres gestantes ou puérperas e seus bebês, possibilitando o planejamento adequado dos fluxos de encaminhamento, da atenção ao parto e aumento da vigilância direcionada à prevenção de óbitos (Menezes et al., 2020).

Verifica-se a importância da testagem universal em gestantes e parturientes visto que a real proporção de casos registrados é fundamental para o planejamento estratégico do cuidado obstétrico e neonatal.

Sobre isso, a pesquisa de Takemoto et al. (2020) mostra números mais elevados de mortes maternas por COVID-19 do que os apresentados nos boletins epidemiológicos no presente estudo,no mesmo período. Essa disparidade pode estar relacionada à inclusão ou não de mortes de puérperas nos índices. Essa inconsistência dos números pode prejudicar o planejamento de ações e não demonstrar a realidade dos fatos, essencial para que se conheça mais detalhadamente os efeitos da doença em gestantes e puérperas.

A inclusão de gestantes e puérperas no grupo de risco para COVID-19 se deve ao número de casos registrados, sendo de grande importância a publicação e fidedignidade das ocorrências. Sobre isso, o estudo de Badr, et al. (2020), que comparou mulheres grávidas infectadas com COVID-19 com mulheres não grávidas, expressou que gestantes apresentaram maior risco de admissão na UTI do que não gestantes. Além disso, demonstraram maior risco de admissão hospitalar devido à descompensação respiratória do COVID-19, tal como dispneia e hipoxemia, para a necessidade de oxigenioterapia, e para intubação endotraqueal.

Sobre a gravidade em gestantes, o estudo de caso realizado por Hantoushzadeh et al. (2020) verificou que mulheres no segundo ou terceiro trimestre de gravidez com infecção por SARS-CoV-2 tem o risco maior de apresentar complicações cardiopulmonares e evoluir para óbito do que não grávidas.

Com o registro do crescimento dos números de casos, gravidade e mortes maternas por COVID-19, viu-se a necessidade de criação e publicação de protocolos com recomendações para prevenção e controle de infecções pelo novo coronavírus para a atenção à gestantes e puérperas. Com isso, em abril de 2020, o Ministério da Saúde emitiu as diretrizes para a manutenção do atendimento pré-natal, parto e puerpério, durante a pandemia, entendendo os riscos que o vírus pode causar nestes grupos (Brasil, 2020a).

Sobre o pré-natal, o Manual de Recomendações para a Assistência à Gestante e Puérpera frente à Pandemia de COVID-19 pontua que, durante o período da pandemia pelo Coronavírus, a assistência deve ser garantida a todas as gestantes (Brasil, 2020a). Além disso, o Ministério da Saúde recomenda que haja a organização dos fluxos de atendimento de gestantes e puérperas na Pandemia de SARS-CoV-2 para proteção de pacientes, contactantes, recém-nascido e profissionais da saúde, com a adoção de fluxo ideal para atendimento, com o objetivo de reduzir o risco de contaminação, orientando e reforçando acerca dos sintomas e cuidados de higiene que devem ser mantidos dentro e fora do ambiente familiar (Brasil, 2020b).

Apesar das recomendações, observou-se que as consultas pré-natais foram suspensas nas Unidades de Saúde de atuação da Residência de Enfermagem Obstétrica durante o início da pandemia, inclusive com suspensão das atividades de residentes nestes estabelecimentos de saúde. Esse período foi variável nos locais de atenção à saúde, sendo de dois a quatro meses sem atendimento.

Segundo Fagundes et al. (2020), a emergência da pandemia de COVID-19 multiplicou as dúvidas e angústias, com o adiamento de consultas e exames, e as medidas de distanciamento social adotadas para conter o avanço da doença.

Essa suspensão das atividades nos estabelecimentos de saúde da Atenção Básica foi contrária às recomendações dos órgãos de saúde, que indicaram a manutenção das consultas de pré-natal com espaçamento entre as elas e intervalo de tempo levando em consideração a idade gestacional, a presença ou não de doenças maternas ou fetais, comorbidades e a evolução da gestação (Brasil, 2020a). 
Segundo o Manual de recomendações para assistência à Gestante Puérpera frente à Pandemia de COVID-19, lançado pelo Ministério da Saúde (Brasil, 2020a), as consultas de pré-natal devem seguir o seguinte fluxo de consultas: entre a $11^{\mathrm{a}}$ e a $14^{\mathrm{a}}$ semana; entre a $20^{\mathrm{a}}$ e a $22^{\mathrm{a}}$ semana; entre a $26^{\mathrm{a}}$ e a $28^{\mathrm{a}}$ semana; 32 semanas; 35 semanas; 37 semanas (quando será colhido RT-PCR para SARS-CoV-2 nos locais e que o resultado do teste demorar mais que 7 dias); 39 semanas e semanais até o parto.

Seguindo a norma técnica da Secretaria de Saúde do Estado do Pará (SESPA, 2020), determina a não paralisação das consultas pré-natal e a marcação de consultas de risco habitual e de alto risco com flexibilização para manter o número adequado de consultas. Ressaltando que tal flexibilização só deve ser aplicada para gestação sem intercorrências, pois gestantes de alta vulnerabilidade e/ou com dificuldade de entendimento das orientações deverão ter o calendário habitual de seguimento pré-natal mantido.

O não atendimento às gestantes pode implicar na detecção tardia de problemas na gravidez (como diabetes gestacional ou estados hipertensivos) ou no feto (como restrições de crescimento intrauterino), trazendo, portanto, riscos maternos e fetais (Brasil, 2020a).

Entretanto, vale ressaltar que foram inúmeros os motivos que levaram à suspensão das atividades pelos profissionais de saúde. Dentre eles, o aumento gradativo do número de casos e taxa de mortalidade dos profissionais de enfermagem, principalmente por conta das más condições de trabalho, falta de Equipamentos de Proteção Individual (EPI), e a testagem dos profissionais, contribuindo com a incômoda liderança em número de óbitos de profissionais de Enfermagem decorrentes da pandemia de COVID-19 no Brasil (Fagundes et al., 2020).

Acredita-se que os órgãos de saúde não devem apenas realizar recomendações e diretrizes para a atuação dos profissionais de saúde, devem fornecer condições para que os mesmos mantenham suas atividades com segurança e saúde necessárias para o bom atendimento dos usuários. Sobre isso, foi possível identificar número insuficiente de profissionais de saúde e sobrecarga de trabalho em virtude da suspeita, afastamento de adoecimento da equipe de enfermagem, além da falta de EPI's suficientes e adequados e necessidade de adequação da infraestrutura recomendada.

Para Alves et al. (2020), os estados do Brasil estimulam a elaboração de protocolos para o atendimento das mulheres com diagnóstico de COVID-19, e reforça a importância de manter o acompanhamento pré-natal, através de agendamentos com horários pré-estabelecidos. Além de que, cada estado possui uma maternidade de referência, e os casos de mulheres gestantes com sintomas respiratórios são avaliados e, quando necessário, encaminhados para os hospitais de retaguarda para a COVID19.

No entanto, no estado do Pará, provavelmente pela sua dimensão territorial e possível dificuldade de acesso às maternidades da região, no início da pandemia houve diversos casos de gestantes sem gravidade, que poderiam receber atendimento em seus municípios de origem, superlotando leitos de urgência do hospital de referência materno-infantil da capital. Ademais, a falta de infraestrutura adequada e capacitação de profissionais de saúde para o atendimento dessas mulheres, culminando em medo e insegurança, podem ter contribuído para os encaminhamentos e referências de gestantes e puérperas para a maternidade de Belém.

Seguindo a norma técnica do SESPA (2020), gestantes com sintomas leves, sem queixas obstétricas associadas, não necessitam ser encaminhada a referência hospitalar. As gestantes devem ser orientadas a manter ao uso de máscara, repouso, hidratação, boa alimentação, manter isolamento social, e o profissional de saúde deverá realizar prescrição de sintomáticos. Além disso, a mulher necessita ser orientada acerca dos sinais e sintomas de piora do quadro, para então procurar o serviço de saúde de referência ao tratamento da COVID-19. Portanto, o enfermeiro, como integrante da equipe de saúde, deve estar habilitado a conhecer todos os estágio e sinais de gravidade da COVID-19. Supõe-se que essas medidas poderiam diminuir a exposição da gestante e possivelmente a superlotação dos hospitais de referência obstétrico do Estado do Pará. 
Em contrapartida, não se deve deixar de mencionar as dificuldades enfrentadas pelos enfermeiros que atuam nos interiores do estado, sabe-se que unidades de saúde pertencentes às regiões urbanas costumam ser bem melhores estruturadas, em detrimento às que são de regiões periféricas, com déficit de recursos (exames, medicamentos), profissionais e transporte. (Almada, Silva, Mardock \& Pimentel, 2020).

Pelos motivos citados acima, o profissional da saúde, particularmente o enfermeiro, deve estar apto, capacitado e vigilante, principalmente aos que atuam no interior do estado, ao manejo dos sinais e sintomas da COVID-19 em gestantes dentro do próprio município, de forma rápida e efetiva, para que estas mulheres sejam transferidas apenas se realmente precisarem, e para aquelas que precisam que possam chegar estáveis e em tempo hábil à maternidade. Pode-se acreditar que tais medidas minimizam os riscos de mortalidade materna pelas implicações da pandemia no Pará.

Diante disso, faz-se necessário que o profissional da saúde, saiba reconhecer o agravamento da doença, e intervir no momento oportuno, com objetivo de evitar a evolução do óbito. De acordo com os resultados, à nível nacional, apenas pouco mais da metade das mulheres, que evoluíram a óbito tiveram assistência de Unidade de Terapia Intensiva, não chegando a $100 \%$ as que utilizaram suporte ventilatório avançado.

No Brasil, segundo estudo de Leal (2020) as barreiras de acesso a serviços com atenção especializada e monitoramento inadequado de complicações obstétricas persistem em hospitais, atenção primária e clínicas especializadas. Em consonância às grandes lacunas encontradas no cenário obstétrico brasileiro atual, o estado do Pará, segundo o estudo de Botelho et al. (2015), demonstrou importantes obstáculos que impossibilitam a redução da mortalidade materna, como a carência do planejamento familiar, a assistência pré-natal inadequada, a superlotação no serviço hospitalar e a deficiência de estrutura dos hospitais, que acarretam precariedade no atendimento às gestantes. Refletindo sobre esses aspectos, soma-se a esse panorama, as dificuldades de acesso acarretadas pela pandemia, o déficit de profissionais, além da escassez de informações sobre o Sars-cov-2.

Notadamente, faz-se necessária mudanças dentro dos fluxos de atendimentos em saúde no contexto da pandemia por COVID19, deve-se redefinir as estratégias nos diferentes níveis de atenção, de modo a se criar novos espaços de acesso da população aos serviços de saúde. Em decorrência do risco de disseminação do vírus de pessoa para pessoa, existe o desejo de minimizar visitas desnecessárias a unidades de saúde. Sendo necessário o desenvolvimento de condutas seguras, com o objetivo de reduzir as consultas de pré-natais, bem como desenvolver técnicas para abordar mulheres que estão em casa ou em quarentena (Barton, Saad, \& Sibai, 2020).

Nesse panorama o uso da tecnologia para atendimentos remotos, tais como as modalidades de teleatendimento ganham destaque nesse período (Daumas,Tasco, \& Costa e Leite,2020). Assim como no estudo de Fryer et al.(2020), mostrou que para diminuir a taxa de transmissão do COVID-19 e limitar a exposição aos pacientes, serviços de saúde na Flórida (e em todo o país) estão fazendo uma rápida transição para a telessaúde. O uso de um modelo de atendimento virtual permite que os profissionais de saúde reduzam as visitas presenciais e incorporem as visitas virtuais à programação do pré-natal. Entretanto, dentro do período deste estudo, não foi observado o uso dessa estratégia para minimização das idas das gestantes aos atendimentos pré-natal, de baixo ou alto risco.

Diante disso, destaca-se o papel do enfermeiro tanto na Atenção Básica, durante o atendimento pré-natal e puerperal, quanto à nível hospitalar. Deve-se frisar que dentro da atenção primária à saúde, aos cuidados à saúde da gestante devem-se incluir orientações, desmistificação de algumas ideias sem comprovações científicas e medidas de prevenção contra a COVID19, tais como a higiene das mãos e das superfícies, o distanciamento social e o uso e confecção de máscaras (Estrela, Silva, Cruz \& Gomes, 2020).

Importante ressaltar que os dados contidos nos boletins epidemiológicos, mostraram que os casos de hospitalizações/complicações por COVID-19 estiveram associados a comorbidades, em consonância com esses dados, o artigo 
de Takamoto (2020), identificou diabetes, doenças cardiovasculares e obesidade como condições significativas associadas à mortalidade na população obstétrica, no contexto da pandemia. Tal fato deve ser ressaltado demonstrando da importância do pré-natal, no manejo dessas doenças, dentro desse contexto pandêmico, o que demonstra a importância do profissional enfermeiro nesse cenário, a fim de se obter menores taxas de mortalidade materna, especialmente por COVID-19.

A Organização Pan-Americana de Saúde (OPS), através de seu boletim de alerta epidemiológico (OPS, 2020) solicita redobramento de esforços para garantir o acesso aos serviços de atenção pré-natal, bem como implementação de medidas preventivas para reduzir a morbidade e mortalidade associadas a COVID-19 em todos os níveis do sistema de saúde, mantendo os objetivos de redução da mortalidade materna e perinatal, dentro do sistema obstétrico mundial.

Nesse contexto, em 22/08/2020 obtém-se a portaria 2.222/2020/GM/MS (Brasil, 2020c), que institui, em caráter excepcional e temporário, Ações Estratégicas de Apoio à Gestação, Pré-Natal e Puerpério e o incentivo financeiro federal de custeio para o enfrentamento da Emergência em Saúde Pública de Importância Nacional (ESPIN) decorrente da pandemia do coronavírus. Tal portaria inclui estratégias para qualificação de ações de atenção ao pré-natal, parto e puerpério, traçando como alguns dos objetivos a otimização dos contatos presenciais e utilização de teleconsultas como recurso complementar; o aprimoramento da triagem clínica para sintomas gripais e suspeitos de COVID-19, sem deixar de investigar as demandas decorrentes da gestação e puerpério; organizar o fluxo interno de acolhimento, identificação, classificação de risco nos casos de atendimento de síndrome gripal, síndrome respiratória aguda grave e suspeita de COVID-19; assim como assegurar a definição de fluxos de referência e contrarreferência para assistência e acompanhamento da mulher durante todo o ciclo gravídico puerperal, considerando as recomendações para os casos suspeitos e confirmados de COVID-19, de acordo com a gravidade do caso, idade gestacional e critérios clínicos para internação em UTI.

Nessa perspectiva, ressaltamos a importância do enfermeiro que presta a assistência obstétrica no Estado do Pará, diante de todas as peculiaridades e dificuldades enfrentadas na região, é necessário que este profissional esteja alerta e seja consciente dos manejos adequados do ciclo gravídico-puerperal em todos os níveis da assistência. Devendo estar atuando da prevenção da infecção à precaução e condutas diante dos agravos, que podem levar ao óbito materno.

\section{Conclusão}

Deste modo, deve-se refletir os desafios que o profissional enfermeiro deve enfrentar e superar ao vivenciar a pandemia da COVID-19 no que diz respeito à sua assistência obstétrica. Nesse contexto, o enfermeiro necessita repensar sua atuação de modo a amenizar ou impedir os impactos da doença para o binômio mãe-filho, pensando estratégias de cuidado que proporcionem bem-estar, tratamento adequado e segurança às mulheres durante todo o período gravídico-puerperal, seja na assistência na atenção primária, secundária ou terciária.

É válido ressaltar que para o enfermeiro atuar de forma efetiva, prestando uma assistência de qualidade, torna-se imprescindível que este conte com subsídios de proteção e infraestrutura para tal, sendo-lhes fornecidos os EPI’s adequados para segurança das gestantes e dos profissionais de saúde.

Estudos sobre a prática da enfermagem obstétrica para COVID-19 devem ser fomentados, uma vez que o enfermeiro atua em todos os níveis da rede de assistência às gestantes. Este profissional necessita estar atualizado acerca de estudos recentes, uma vez que há escassez de pesquisas, e seguir as normativas e recomendações sobre os fluxos de atendimentos, com a intenção de contribuir parar a redução da mortalidade materna por COVID-19.

Pesquisas e trabalhos científicos futuros devem explorar ainda mais a temática, pois no que tange a COVID-19 no período gravídico-puerperal são ainda mais insipientes. Deve-se reforçar as diferenças entre o acometimento e gravidade da doença em mulheres gravidas e população em geral, fazendo a identificação de quadros mais graves de maneira rápida e eficaz, 
reforçando os parâmetros de avaliação existentes e treinando profissionais de saúde para atuar de forma diferenciada frente a gestantes que apresentem sintomas de alerta.

\section{Referências}

Almada, L. C. L. de., Silva, C. de A., Mardock, A. R. M., Pimentel, Z. N. De S. (2020). Desafios da assistência pré-natal em um município no interior da Amazônia. Saúde em Redes; 6(2):1124.

Alves, V. H., Souza, K. V. de., Carmo, J. M. A. do., Moretto, V. L., Teixeira, R. C., Freitas, W. de M. F., \& Sousa, E de L. C. de. (2020). Enfermagem obstétrica e sua força de trabalho em tempos de COVID-19: relato de experiência das regiões do brasil. Enferm. Foco; 11, 103-108.

Badr, H. S., Du, H., Marshall, M., Dong, E., Squire, M. M., \& Gardner, L. M. (2020). Association between mobility patternsand COVID-19 transmission in the USA: a mathematica lmodelling study. The Lancet. Infectiousdiseases, 20(11), 1247-1254. https://doi.org/10.1016/S1473-3099(20)30553-3

Barton, J. R., Saade, G. R., \& Sibai, B. M. (2020). A Proposed Plan for Prenatal Care to Minimize Risks of COVID-19 to Patients and Providers: Focus on Hypertensive Disorders of Pregnancy. American journal of perinatology, 37(8), 837-844.

Botelho, N. M., Silva, I. F. M. M., Tavares, J. R., \& Lima, L. O. (2014). Causas de morte materna no Estado do Pará, Brasil. Revista Brasileira de Ginecologia e Obstetrícia, 36(7), 290-295. https://doi.org/10.1590/SO100-720320140004892

Daltro, M. R., \& Faria, A. A. Relato de experiência: Uma narrativa científica na pós-modernidade. Estud. pesqui. psicol., 19(1), 223-237, 2019.

Daumas, R. P., Silva, G. A., Tasca, R., Leite, I. C., Brasil, P., Greco, D. B., Grabois, V., \& Campos, G. W. de S. (2020). O papel da atenção primária na rede de atenção à saúde no Brasil: limites e possibilidades no enfrentamento da COVID-19. Cadernos de Saúde Pública,36(6), e00104120. https://doi.org/10.1590/0102-311x00104120

Estrela, F. M., Silva, K. K. A., Cruz, M. A., \& Gomes, N. P.(2020). Gestantes no contexto da pandemia da COVID-19: reflexões e desafios. Physis. 30(2), e300215. https://doi.org/10.1590/s0103-73312020300215.

Fagundes, M. C. M., Alves, V. H., Bonazzi, V. C. A. M., Sampaio, M. do R. de F. B., Sousa, E.de L. C. de., Rodrigues, D. P., Pinheiro, V. E., \& Freire, N. P. (2020). Anseios das profissionais de enfermagem gestantes frente à pandemia de COVID-19: um relato de experiência. Enferm. Foco, 11 (Esp. 2 ): 109-113.

Fryer, K., Delgado, A., Foti, T., Reid, C., \& Marshall, J. (2020). Implementation of Obstetric Telehealth During COVID-19 and Beyond. Maternal andChild Health Journal. 24(9):1104-1110. https://link.springer.com/article/10.1007/s10995-020-02967-7

Governo do Estado do Pará. Secretaria de Estado de saúde Pública do Pará - SESPA (2020). Nota técnica nº $1 / 2020$. Dispõe sobre orientações ao atendimento de gestantes e puérperas no plano de contingência ao COVID-19. Coordenação Estadual de Saúde da Mulher. PA: Secretaria de Estado de saúde Pública.

Hantoushzadeh, S., Shamshirsaz, A. A., Aleyasin, A., Seferovic, M. D., Aski, S. K., Arian, S. E., Pooransari, P., Ghotbizadeh, F., Aalipour, S., Soleimani, Z., Naemi, M., Molaei, B., Ahangari, R., Salehi, M., Oskoei, A. D., Pirozan, P., Darkhaneh, R. F., Laki, M. G., Farani, A. K., Atrak, S., \& Aagaard, K. (2020). Maternal deathdueto COVID-19. American journalofobstetricsandgynecology, 223(1), 109.e1-109.e16. https://doi.org/10.1016/j.ajog.2020.04.030

Leal, M. do C., Esteves-Pereira, A. P., Viellas, E. F., Domingues, R. M. S. M., \& Gama, S. G. N. da. (2020). Prenatal care in the Brazilian public health services. Revista de Saúde Pública, 54, 08. https://doi.org/10.11606/s1518-8787.2020054001458

Li, T. (2020). Diagnosis and clinical management of severea cute respiratory syndrome Coronavirus 2 (SARS-CoV-2) infection: an operation alrecommendation of Peking Union Medical College Hospital (V2.0): WorkingGroupof 2019 Novel Coronavirus, Peking Union Medical College Hospital. EmergingMicrobesandinfections, 9(1). https://doi.org/10.1080/22221751.2020.1735265

Menezes, M. de O., Andreucci, C. B., Nakamura-Pereira, M., Knobel, R., Magalhães, C. G., \& Takemoto, M. L. (2020). Testagem universal de COVID-19 na população obstétrica: impactos para a saúde pública. Cadernos de Saúde Pública, 36(8), e00164820. https://doi.org/10.1590/0102-311x00164820

Ministério da Saúde (2020a). Manual de recomendações para a assistência à gestante e puerpéria frente à pandemia de COVID-19. Secretaria de Atenção Primária à Saúde: Ministério da Saúde.

Ministério da Saúde (2020b). Nota Técnica No 12/2020 COSMU/CGCIVI/DAPES/SAPS/MS. Dispõe sobre a Infecção COVID-19 e os riscos às mulheres no ciclo gravídico-puerperal. Secretaria de Atenção Primária à Saúde: Ministério da Saúde.

Ministério da Saúde. Gabinete do Ministro. (2020c). Portaria $N^{o}$ 2.222/GM/MS, de 25 de agosto de 2020. Institui, em caráter excepcional e temporário, Ações Estratégicas de Apoio à Gestação, Pré-Natal e Puerpério e o incentivo financeiro federal de custeio para o enfretamento da Emergência em Saúde Pública de Importância Nacional (ESPIN) decorrente da pandemia do coronavírus. Diário Oficial da União, Brasília, DF, p. 49.

Organización Panamericana de la Salud (OPS) (2020). Alerta Epidemiológica: COVID-19 en el embarazo: OPS/OMS.

Takemoto, M., Menezes, M. O., Andreucci, C. B., Nakamura-Pereira, M., Amorim, M., Katz, L., \& Knobel, R. (2020). The tragedyof COVID-19 in Brazil: 124 maternal deathsandcounting. Internationa ljournal of gynaecology and obstetrics: the official organ of the International Federation of Gynaecology and Obstetrics, 151(1), 154-156. https://doi.org/10.1002/ijgo.13300 